Original Research

\title{
Single-Case Neuropsychological Assessment of a Patient with a Posterior Parietal Lesion Using Behavioral Testing and Resting-State fMRI
}

Elisa Martín-Arévalo ${ }^{1,2,3,{ }^{\dagger}, \text { Carole Guedj }}{ }^{2,4,+}{ }^{+}$, François Cotton ${ }^{1,2,5}$, Gilles Rode ${ }^{1,2,6}$, Karen T. Reilly 1,2 , Fadila Hadj-Bouziane $2,4,+,{ }^{*}$, Laure Pisella ${ }^{1,2,+, *}$

1. Trajectory team, INSERM U1028, CRNS-UMR5292, Lyon Neuroscience Research Center (CRNL), Lyon, France; E-Mails: emartina@ugr.es; francois.cotton@univ-lyon1.fr; gilles.rode@univIyon1.fr; karen.reilly@inserm.fr; laure.pisella@inserm.fr

2. University UCBL Lyon 1, University of Lyon, Lyon, France; E-Mails: Carole.Guedj@unige.ch; fadila.hadj-bouziane@inserm.fr

3. Department of Experimental Psychology and Brain, Mind, and Behavior Research Center (CIMCYC), University of Granada, Spain

4. Impact team, INSERM U1028, CRNS-UMR5292, Lyon Neuroscience Research Center (CRNL), Lyon, France

5. MRI center, Centre Hospitalier Lyon Sud, Hospices Civils de Lyon, Pierre Bénite, France; Observatoire Français de la Sclérose en Plaques, Lyon, France; CREATIS-LRMN, CNRS/UMR/5220INSERM U630, Villeurbanne, France

6. Service de Rééducation Neurologique, Hôpital Henry Gabrielle, Hospices Civils de Lyon, France

¥ Current Affiliation: Department of Fundamental Neuroscience, Laboratory for Behavioral Neurology and Imaging of Cognition, University Medical School of Geneva - Campus BIOTECH H8, 9 Chemin des Mines, 1202, Geneva, Switzerland.

$\dagger$ These authors contributed equally to this work.

* Correspondence: Laure Pisella and Fadila Hadj-Bouziane; E-Mails: laure.pisella@inserm.fr; fadila.hadj-bouziane@inserm.fr

Academic Editor: Jesper Mogensen

Special Issue: Brain and Visual Processing

OBM Neurobiology

2021, volume 5, issue 3
Received: January 29, 2021

Accepted: August 12, 2021

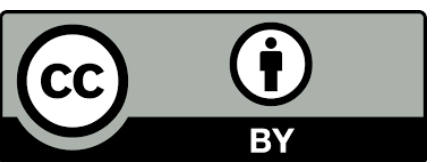

(C) 2021 by the author. This is an open access article distributed under the conditions of the Creative Commons by Attribution License, which permits unrestricted use, distribution, and reproduction in any medium or format, provided the original work is correctly cited. 


\begin{abstract}
This study integrated functional connectivity measures using resting-state $\mathrm{FMRI}$ and behavioral data from a single-case observation of patient (PER) one year after righthemispheric hemorrhage in the intraparietal sulcus and superior parietal lobule (IPS/SPL). PER showed no sign of clinical neglect. Her behavioral performance in the visuo-manual pointing task and in the letter discrimination task under conditions of endogenous and exogenous attentional cueing was compared between the left (affected) and right (unaffected/control) peripheral visual fields. The resting-state $\mathrm{fMRI}$ demonstrated an imbalance between the right and left hemispheric frontoparietal functional connectivity within the dorsal attentional and motor networks. Although the frontal and occipital cortices were not structurally damaged, specific fronto-occipital functional connectivity was imbalanced, which was strongly associated with the behavioral changes. First, the activity in the right frontal eye field showed weaker correlations with the activity in the right inferior occipital area compared to the correlation with the activity in the left inferior occipital area. This imbalanced fronto-occipital functional connectivity was accompanied by a specific impairment in endogenous covert attention in the left visual field. Second, the activity within M1 in both hemispheres showed weaker correlations with the activity of the right cuneus compared to the correlation with the activity in the left cuneus. The imbalanced fronto-occipital functional connectivity was associated with the impairment of the reaching movement of the left and right hands towards the left visual field (optic ataxia). Altogether, our results showed that a lesion to the posterior parietal cortex affects the relationship between distal regions underlying the sensorimotor and attentional abilities.
\end{abstract}

\title{
Keywords
}

Optic ataxia; resting-state fMRI; frontoparietal networks; fronto-occipital networks; endogenous attention; exogenous attention

\section{Introduction}

Covert attention facilitates the perception of peripheral visual field stimuli and relies on topdown modulation of areas by frontoparietal attentional networks within the occipitotemporal visual stream dedicated to object recognition. For example, pre-saccadic attentional facilitation involves a functional network within which the frontal eye fields (FEFs) modulate activity in the inferior occipital area V4 [1-8]. Two main frontoparietal attentional networks have been described, the dorsal network and the ventral network. The dorsal frontoparietal attentional network - (DAN) [9, 10] is bilateral and includes the FEFs and regions of the intraparietal sulcus and the superior parietal lobule (IPS/SPL). The ventral frontoparietal attentional network - (VAN) $[9,10]$ is restricted to the right hemisphere and includes two frontal regions, the medial frontal gyri (MFG) and the inferior frontal gyri (IFG), as well as a parietal region known as the supramarginal gyrus (SMG), which is a part of the temporoparietal junction (TPJ). Attentional disorders, for example, clinical spatial neglect, 
have been associated with lesions in the right TPJ (VAN) [11, 12] but not with exclusive lesions in the IPS/SPL (DAN). Instead, clinical examination of patients with IPS/SPL lesions often reveals optic ataxia, a neurological deficit in which patients have no primary perceptual or motor deficits but present difficulties in responding to objects in the contralesional peripheral visual field [13-20], and in fast visuomotor transformations known to depend on interactions between the occipito-parietal region and the dorsal premotor and primary motor cortices (e.g., [21-30]). Interestingly, close examination of patients with focal IPS/SPL lesions and without clinical neglect syndrome, for example, by using briefly flashed stimuli, sometimes reveals attentional difficulties in the form of sub-clinical visual detection and discrimination deficits that are specific to covert endogenous orienting in the contralesional peripheral visual field [31-36].

According to the classical neuropsychological approach, the site of structural damage is associated with a clinical behavioral deficit [37]. By complementing this approach with functional magnetic resonance imaging ( $\mathrm{FMRI}$ ), it is possible to better understand the consequences of the structural damage at the level of brain networks [38]. This is particularly interesting because structural damage to a different node of a network can have different functional consequences on the rest of the network, and these consequences are not necessarily reciprocal. For example, a study found that damage to the right inferior frontoparietal regions (VAN) induced an interhemispheric imbalance within the DAN that affected the patient's attentional performance [39]. Thus, a focal lesion within the posterior parietal cortex can have functional consequences on other structurally intact parietal regions. Conversely, another study [34] showed that unilateral damage to the right IPS/SPL (DAN) did not alter the resting-state connectivity of the right inferior frontoparietal regions (VAN). However, a focal lesion within the posterior parietal cortex might have functional consequences on the interaction between other non-damaged nodes of the attentional or sensorimotor network, such as affecting the functional relationship between the structurally intact frontal and occipital regions. Determining the effect of lesions in the posterior parietal cortex on the sensorimotor and attentional abilities and the activity within brain networks is thus important.

Previously, we documented a lesion in the IPS/SPL region in a patient (PER), with optic ataxia in the left (contralesional) visual field and no clinical neglect [18]. Here, we report the exogenous and endogenous covert attentional abilities of the patient and the resting-state functional connectivity. All tests were performed in the same week as those that were performed to assess the visuomotor abilities reported by Blangero et al. [18]. Brain networks underlying specific functions, such as attention (DAN/VAN) or sensorimotor integration, can be identified at rest in the absence of any explicit task or stimuli by measuring the temporal correlation - (functional connectivity) - of the blood oxygenation level-dependent signal using functional magnetic resonance imaging (fMRI; [4042]). We assessed the integrity of the brain networks underlying attentional and sensorimotor integration and determined the relationship between the integrity of these networks and the patient's attentional and visuomotor behavior. We assessed potential proximal effects of the lesion by examining the functional connectivity at rest between frontal regions and the preserved, perilesional part of the parietal cortex. Additionally, we assessed potential distal effects by examining the functional connectivity of specific fronto-occipital interactions related to visuomotor and attentional deficits displayed by the patient at rest. 


\section{Materials and Methods}

\subsection{Case Report}

Patient PER, a right-handed female, was 70 years old when she suffered a hemorrhagic stroke in the right hemisphere. At the time of the examination (one year after the stroke), we performed anatomical MRI, resting-state $\mathrm{FMRI}$, and behavioral examinations. The occipital, temporal, and frontal lobes, as well as the postcentral gyrus, were largely unaffected. The lesion (bottom-right panel of Figure 1) involved the posterior parietal cortex and, more specifically, the IPS/SPL region (see 18). The lesion extended into the deep white matter and included parts of the angular and the supramarginal gyri but spared most of the temporoparietal junction (TPJ: yellow area, stimulusdriven attention network on the figure adapted from Corbetta and Shulman [43], the bottomcenter panel of Figure 1). Patient PER transiently showed a visual neglect syndrome in the acute phase. While testing in the chronic phase, the patient exhibited optic ataxia when using either hand in her left visual field (field effect) and with her left hand in both hemifields (hand effect) but showed no signs of apraxia or clinical neglect during standard neuropsychological tests (cancellation and drawing tasks, [18]).



Figure 1 Anatomical localization of patient PER's lesion. The bottom-right figure illustrates a lateral view of the cortical surface with PER's lesion shown in black (T1 anatomical MRI). The colored parts superimposed on it in the image above represent regions of the parietal cortex based on the automated anatomical labeling ( $A A L)$ parcellation [44] (Parietal_Superior: cyan; Parietal_Inferior: dark blue; SupraMarginal: yellow; Angular: green; Precuneus: red). The white insert (bottom-left) shows the posterior parietal cortex of the dorsal stream (according to Milner and Goodale $[15,17]$ ), the Dorsal and Ventral Attentional networks (according to Corbetta and Shulman [43]), and the cortical areas damaged due to spatial neglect (according to Corbetta and Shulman [43]). The images at the top illustrate PER's lesion (outlined by a white dotted line) mapped on the AAL parcellation (colored areas) on coronal sections from posterior to anterior parts of the parietal lobe (left to right). 


\subsection{Behavioral Procedures}

\subsubsection{Experiment 1: Reaching during Fixation (Pro-Reaching)}

Details of the apparatus, procedure, and the data for this task are published in Blangero et al. [18] (see Figure 2A for an illustration of the experiment). Briefly, each trial began with the illumination of the starting position of the hand at the bottom of the tactile screen. Upon detection of the correct position of the hand on the screen, an eccentric fixation point (white cross) appeared. Six fixation positions were relative the screen, aligned horizontally (at $30^{\circ}, 25^{\circ}, 20^{\circ}$ left and $20^{\circ}, 25^{\circ}$, $30^{\circ}$ right). After the system detected that the eyes were at the correct position and remained there for $500 \mathrm{~ms}$, a reaching target (closed white circle) was illuminated for $800 \mathrm{~ms}$ at the centre of the screen. Patient PER was asked to maintain gaze on the fixation position during the presentation of the peripheral reaching target. The target was then extinguished. After a $500 \mathrm{~ms}$ delay, the heard an auditory tone, which indicated that she had to reach to the remembered target position while maintaining fixation. There were five repetitions per target position. The target locations were randomly presented across the six possible locations. The mean and the confidence ellipse (95\%) of the movement endpoints were computed for each target position in the right and left visual fields (Figure 2).


Figure 2 Illustration of the pro-reaching task (reaching to peripheral targets while keeping the eyes on a central fixation cross) and 2D illustration of PER's reaching endpoints while pointing with her right (ipsilesional) hand to target locations symbolized by black squares. Each movement endpoint (empty circles), the mean endpoint (filled circles), and the 95\% confidence ellipses are shown for each target position in the right and left visual fields (adapted from Blangero and colleagues, [18]). 


\subsubsection{Experiment 2: Letter Discrimination Tasks (See Figure 3).}

Endogenous and exogenous covert attention conditions were defined according as per Chica et al. [45]. For the trials with the endogenous condition, we used the experimental setup that was used to assess another patient reported in Blangero et al. [32] (Figure 3). Briefly, five target locations were presented as symbols of " 8 " on both sides of a central visual fixation point for a random period between 1 and $2 \mathrm{~s}$. Eye position was monitored online using eye-tracker recordings (Cambridge Research System, Rochester, UK. Frequency: $250 \mathrm{~Hz}$ ) and patient PER kept her eyes on the fixation point throughout the task. If a saccade was initiated, the trial was automatically replayed. While the eyes were fixated, a central cue - (green arrow) - was presented for $150 \mathrm{~ms}$, which indicated the location (green) and the direction (left or right) of the target. The target letter always appeared at the green position ( $8^{\circ}$ eccentricity) in the left or the right visual field (the presentation side was randomized). The target letter was presented for $250 \mathrm{~ms}$ and then masked by the reappearance of the " 8 " symbols. The directional central cue always indicated the upcoming target location correctly. At the end of each trial, the subject was asked to press one of two buttons (with no time constraints) to indicate whether the letter presented was $E$ or inverted-E (two-alternative forced-choice paradigm). The trials with the exogenous condition used a similar setup as those with the endogenous condition, except that the cue was peripheral and not central. The position of the green cue $\left(8^{\circ}\right.$ eccentricity) was flashed for $150 \mathrm{~ms}$ in either the left or the right visual field, which indicated the location of the upcoming target for all trials. 


\section{Exogenous Condition}

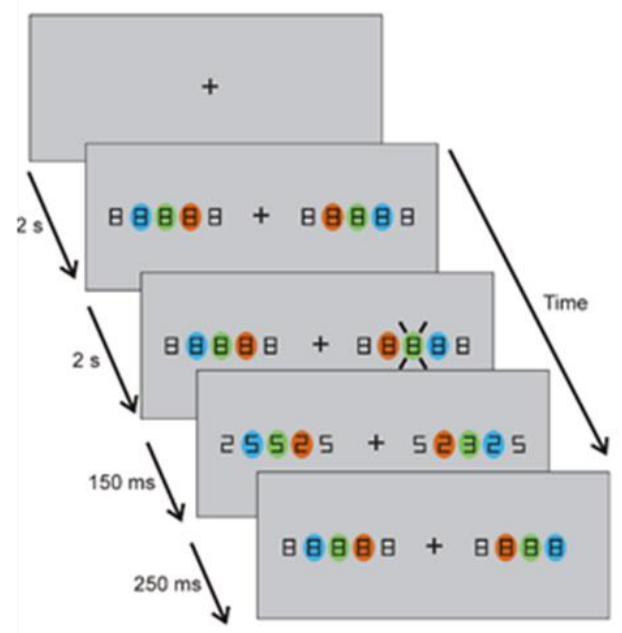

Endogenous Condition

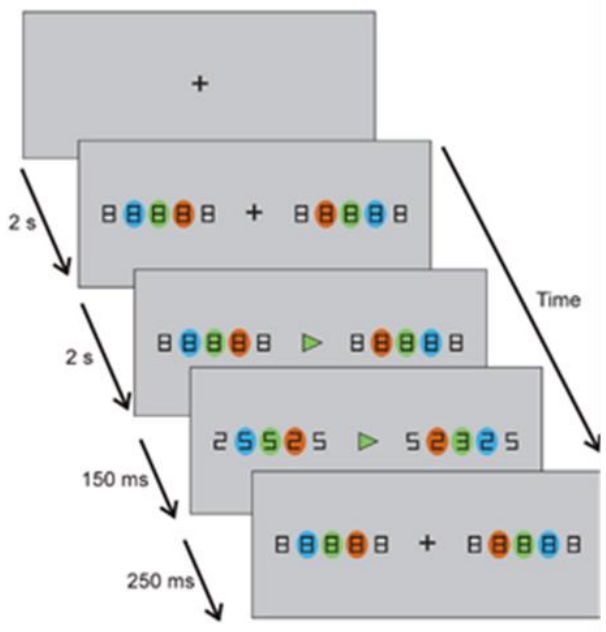

$$
x^{2}(1)=25
$$

$$
\begin{aligned}
& x^{2}(1)=25 \\
& p<.0001
\end{aligned}
$$

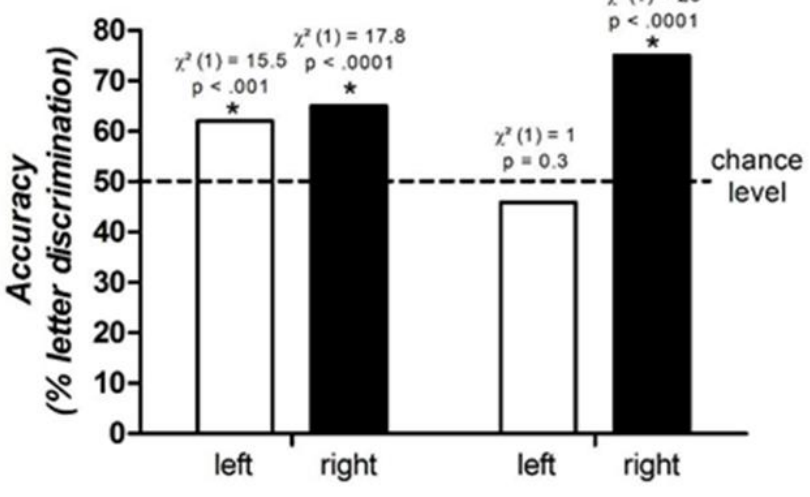

Exogenous

Figure 3 Letter discrimination tasks. Top panel: Illustration of the two experimental conditions. The green cues were always active ( $8^{\circ}$ eccentricity) either in the right or the left visual field. The exogenous cue consisted of a peripheral green flash on one side ( shown here with diagonal lines, representing the location of the green flash in the right hemifield). The endogenous cue consisted of a green central arrow that informed the target location through direction and color. In both conditions, the letter $E$ was flashed for $250 \mathrm{~ms}$ in regular or inverted orientation at the location of the cue, surrounded by flankers that also changed from a symbol of " 8 " to either " 2 " or " 5 " for $250 \mathrm{~ms}$. After $250 \mathrm{~ms}$, all symbols were masked by the reappearance of the " 8 " symbols. Bottom panel: PER's performance in the letter discrimination task, presented in the visual periphery at $8^{\circ}$ eccentricity in the left and right visual fields under conditions of peripheral (exogenous) versus central (endogenous) cueing.

Patient PER participated in 60 trials across four sessions for both the exogenous and endogenous conditions, starting with two sessions of the exogenous condition followed by two sessions of the endogenous condition on the first day. On the second day, the order of presentation of the trials was reversed, but the number of trials and sessions was the same. The accuracy, or the percentage of correct letter discrimination, was computed based on 120 trials per condition (central cueing/endogenous - or peripheral cueing/exogenous) and side (left - or right). Chi- squared tests 
were performed to determine whether the accuracy was above the level of chance $(50 \%)$ with alpha $=0.001$.

\subsection{Imaging Procedures}

Imaging data were collected on a 3T Philips scanner. A spoiled GRASS sequence was used to obtain an anatomical scan; the in-plane resolution was $0.5 \times 0.5 \mathrm{~mm}$ and the slice thickness was 0.9 $\mathrm{mm}$. A gradient echo sequence (TR $=3 \mathrm{~s} ; 1.8 \times 1.8 \times 6 \mathrm{~mm}$; 157TRs) was used to obtain a functional run from the whole brain while PER was resting in the dark (with no music); she was allowed to close her eyes. Data were preprocessed using the AFNI software [46].

\subsubsection{Anatomical Scan and Determination of the Extent of the Lesion}

The anatomical scan of the patient was co-registered with the automated anatomical labeling (AAL) parcellation [44]. The AAL is a digital human brain atlas with 90 labeled volumes. A mask was manually drawn around the site of the lesion. As shown for the coronal MRI slices overlaid on the AAL atlas (top panels of Figure 1), the lesion occurred in the superior parietal lobule (SPL-according to the AAL atlas, marked in - cyan; $74 \%$ of voxels within the region labeled as "parietal superior" were damaged) and the intra-parietal sulcus (IPL-in the AAL atlas, - marked in dark blue; 54\% of voxels within the region labeled "parietal inferior" were damaged), corresponding to the IPS/SPL region of the DAN (blue area in the figure; the network for goal-directed attention from Corbetta and Shulman [43] is reproduced at the bottom- center of Figure 1). The lesion extended into the deep white matter and the regions labeled angular gyrus (in the AAL atlas, - marked in green; 65\% of voxels within the region were damaged), supramarginal gyrus (in the AAL atlas, - marked in yellow; $34 \%$ of voxels within the region were damaged) and precuneus (in the AAL atlas, - marked in red; $21 \%$ of voxels within the region were damaged). The parieto-occipital sulcus, which includes the human homolog of V6A [30], was spared.

\subsubsection{Resting-State Functional Connectivity Networks}

The preprocessing pipeline of the functional run included despiking, motion correction, and temporal filtering to extract the spontaneous, slowly fluctuating brain activity $(0.01-0.1 \mathrm{~Hz})$. The functional data were then realigned to the anatomical data and registered on the TT_ ICBM452 template using the 'Ipa' cost function implemented in AFNI. Nuisance variables, such as the six parameter estimates for head motion, as well as the cerebrospinal fluid and white matter signals, were removed by performing a linear regression analysis based on the tissue probability maps created from the patient's anatomical scans using the FAST toolbox [47]. The regression analysis included a manually drawn mask around the lesion to exclude the voxels that were a part of the lesion. Spatial smoothing was performed with an 8-mm FWHM Gaussian kernel and was applied to the output of the regression.

\subsubsection{Frontoparietal and Fronto-Occipital Functional Connectivity: Attentional and Motor Networks}

We used a seed-based approach to measure the functional connectivity of specific welldocumented fronto-occipital relationships within and between the hemispheres, as well as the functional connectivity profiles within the dorsal and ventral frontoparietal attentional and motor 
networks. We selected seeds of 1 voxel within the frontal cortex and used the AAL parcellation [44] to obtain their correlation scores with parietal and occipital subdivisions. The 1-voxel seeds included the signal from neighboring (particularly in-plane) voxels due to the low resolution of the functional images and the particular spatial smoothing filter applied.

Frontal Seed Selection. For the attentional networks, we selected four 1-voxel seeds (two each for VAN and DAN) based on the Montreal Neurological Institute (MNI) coordinates published by Fox et al. [48]. DAN: right and left Frontal Eye Field (FEFr: $x=22, y=-4, z=52$; FEFI; $x=-25, y=-9, z=$ 51) and VAN: right Inferior Frontal Gyrus (IFGr; $x=46, y=38, z=3$ ) and right Middle Frontal Gyrus (MFGr; $x=36, y=37, z=28$ ).

For the motor networks, we selected four 1-voxel seeds (two within the primary motor cortex and two within the supplementary motor area) based on the MNI coordinates published by Grefkes et al. [49]. The right and left M1 (M1r; $x=37, y=-24, z=56 ; M 1$ l; $x=-37, y=-24, z=56)$ within the right and left "postcentral" regions, according to the AAL atlas; right SMA (SMAr; $x=4, y=2, z=55$ ) and left SMA (SMAl; $x=-5, y=-12, z=55$ ) within the left and right "paracentral" lobules according to the AAL atlas.

Parietal and Occipital Region Selection. Five regions of the posterior parietal cortex were included in the analysis based on the AAL subdivision and comprised the superior and inferior parietal lobules (SPL, IPL), the angular, and supramarginal gyri (ANG, SMG), and the precuneus. According to the AAL subdivision, the ANG and SMG correspond to the TPJ (VAN stimulus-driven network), while the SPL and IPL correspond to the goal-directed attention network DAN [43]. Voxels that overlapped with the site of the lesion in the images of the right hemisphere were excluded from all analyses. To assess the potential proximal effects of the parietal lesion, we examined the functional connectivity at rest between each of the eight 1-voxel frontal seeds and the five parietal regions, some of which were perilesional. The final number of voxels included in these analyses is reported in the table included in Figure 4. Furthermore, we added two regions of the occipital cortex in the analysis, which included the Inferior Occipital region and the Cuneus, based on the AAL subdivision. We included the Inferior Occipital region because the MNI coordinates of the human homolog of V4 [6], known to be functionally associated with the FEF for attentional facilitation, were located in this region according to the AAL atlas. The Cuneus was included because the MNI coordinates of the human homolog of V6A [30], known to be functionally linked to dorsal premotor and motor cortices for direct visuomotor transformations, were located between this region and the precuneus according to the AAL atlas. To determine the potential distal effects of the parietal lesion, we examined the functional connectivity at rest between each of the eight 1-voxel frontal seeds and the two occipital regions. The final number of voxels included in these analyses is reported in the table included in Figure 5. 


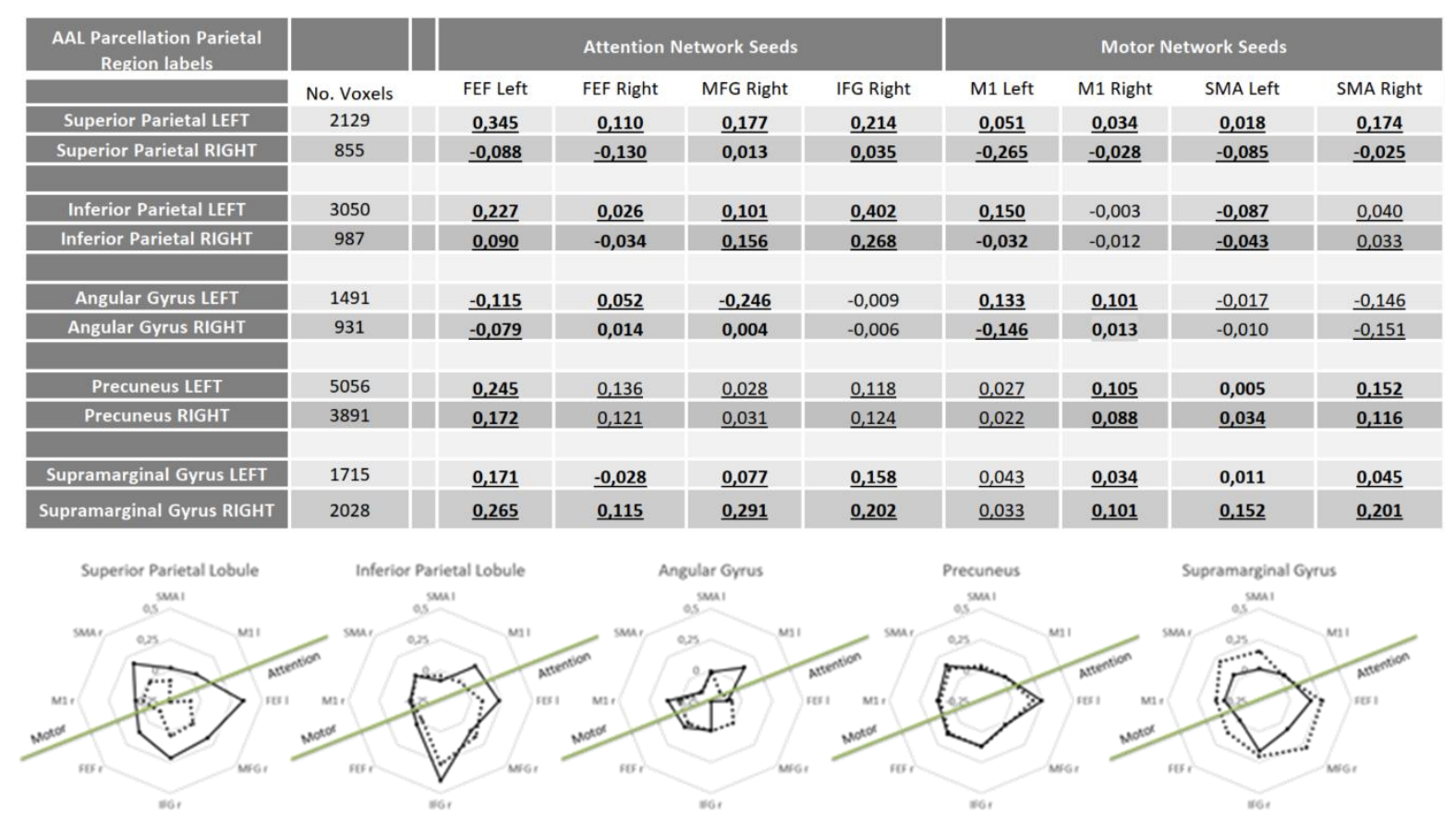

Figure 4 Frontoparietal functional connectivity. Table: Shown are the mean correlation scores between the AAL parietal parcellation regions as per Tzourio-Mazoyer et al. [44], and the eight frontal seeds selected for the attention network based on the study by Fox et al. [48] and for the motor network based on Grefkes et al. [49]. Underlined values indicate correlations that are significantly different from zero. Values in bold indicate that the two hemispheres show significant differences for the correlation between the seed and the AAL region, e.g., the right and left Superior Parietal regions show significant differences for the correlation between the FEF left seed and the AAL region. Diagrams: Shown are the parieto-frontal correlations for the left and right posterior parietal regions (left hemisphere: solid lines; right hemisphere: dashed lines). 


\begin{tabular}{|c|c|c|c|c|c|c|c|c|c|}
\hline \multirow{2}{*}{$\begin{array}{c}\text { AAL Parcellation Occipital } \\
\text { Resionlabels }\end{array}$} & \multirow[b]{2}{*}{ No. Voxels } & \multicolumn{4}{|c|}{ Attention Network Seeds } & \multicolumn{4}{|c|}{ Motor Network Seeds } \\
\hline & & FEF Left & FEF Right & MFG Right & IFG Right & M1 Left & M1 Right & SMA Left & SMA Right \\
\hline Cuneus LEFT & 2158 & $\underline{0,191}$ & $\underline{0,194}$ & $\underline{0,155}$ & $\underline{0,090}$ & $\underline{0,136}$ & $\underline{0,078}$ & $\underline{0,159}$ & $\underline{0,210}$ \\
\hline Cuneus RIGHT & 2174 & 0,234 & $\underline{0,173}$ & $\underline{0,223}$ & $\underline{0,084}$ & $\underline{0,058}$ & $\underline{0,042}$ & $\underline{0,134}$ & $\underline{0,182}$ \\
\hline Inferior Occipital LEFT & 1366 & $\underline{0,216}$ & $\underline{0,234}$ & $\underline{0,070}$ & $\underline{0,090}$ & $\underline{0,162}$ & $\underline{0,197}$ & $\underline{0,183}$ & $\underline{0,222}$ \\
\hline Inferior Occipital RIGHT & 1199 & 0.191 & $\underline{0.081}$ & $\underline{0,072}$ & $\underline{0.094}$ & $\underline{0.161}$ & $\underline{0.233}$ & $\underline{0.115}$ & $\underline{0.095}$ \\
\hline
\end{tabular}
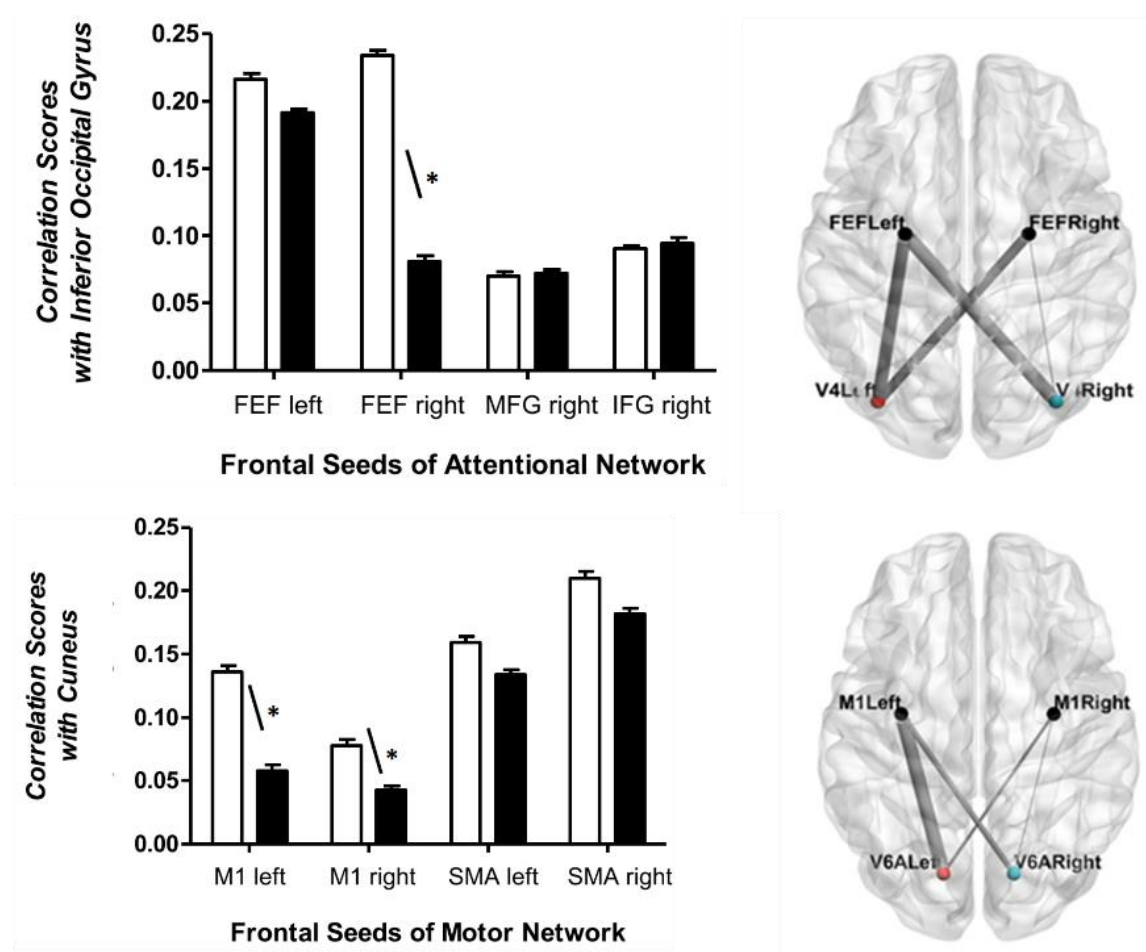

Figure 5 Fronto-occipital functional connectivity. Table: Shown are the correlation scores between the AAL occipital parcellation regions and the eight frontal seeds. Underlined values indicate correlations that are significantly different from zero. Values in bold indicate the two hemispheres have significant differences for the correlation between the seed and AAL region. Diagrams: Top left panel: Shown are the correlations between the frontal seeds of the attentional network and the left (open bars) or right (solid bars) inferior occipital gyrus. Top right panel: Pictorial representation of the strength of the correlation between the left and right FEFs and the left and right inferior occipital gyrus. Thicker lines indicate stronger correlations. Bottom left panel: Shown are the correlations between the frontal seeds of the motor network and the left (open bars) or right (solid bars) cuneus. Bottom right panel: Pictorial representation of the correlation strength between the left and right M1s and the left and right cuneus. Thicker lines indicate stronger correlations. For the left top and bottom panels, bar plots represent mean (+SD)

To minimize the risk of including the information regarding damaged tissue in the final analysis, a mask was manually drawn around the site of the lesion, identifiable from the patient's anatomical scan. Additionally, the lesion mask was included in the regression analysis while determining the correlation. Despite these precautions, it is possible that some damaged tissue, undetected in the anatomical scan, was included in the analysis. 


\subsection{Correlations}

We computed the correlation maps (based on Pearson's correlation test) for each of the eight frontal seeds with the parietal $(n=5)$ and occipital $(n=2)$ regions described in the previous section. To determine the proximal effect of the right parietal lesion on the frontoparietal connectivity, we used unpaired t-tests to compare the correlation scores between a specific frontal seed and all the voxels in each posterior parietal AAL parcellation region in the left and right hemispheres. To determine the distal effect of the right parietal lesion on the fronto-occipital connectivity, we used unpaired t-tests to compare the correlation scores between a specific frontal seed and all the voxels in each occipital AAL parcellation region in the left and right hemispheres. For the attentional network, we were particularly interested in the well- characterized functional interaction between the FEF (DAN) and the inferior occipital gyrus. The functional connectivity between the right IFG and MFG seeds (VAN) and the inferior occipital gyrus was included as a control condition. For the motor networks, we focused on the well- characterized functional interaction between $\mathrm{M} 1$ and the cuneus during visuomotor tasks and determined the correlation between the SMA seeds and the cuneus as a control.

The alpha value was set to 0.001 , and Bonferroni correction was incorporated into the analysis to account for the number of tests performed (168); the $p$-value threshold for all correlations was set at $0.001 / 168=5.9 \times 10^{-6}$.

\section{Results}

\subsection{Behavioral Results}

\subsubsection{Experiment 1: Reaching during Fixation}

As illustrated in Figure 2, patient PER exhibited left optic ataxia, characterized by large hypometric errors when pointing to visual targets within the left (contralesional) visual field (2.92 $\pm 0.73 \mathrm{~cm}$ ), compared to the pointing errors for the right visual field $(0.87 \pm 0.20 \mathrm{~cm})$. These data have been published [18].

\subsubsection{Experiment 2: Letter Discrimination Tasks}

The percentage of correct choices made by the patient for the letter discrimination under exogenous and endogenous conditions is presented in the bottom panel of Figure 3. Chi-square tests revealed that the accuracy was at the chance level only for targets briefly flashed within the left (contralesional) visual field with an endogenous cue (45.9\%). A one-sided Fischer's Exact test showed that the left/right distribution of accuracies significantly depended on the exogenous/endogenous condition (Odds Ratio $=1.59$ [0.93; 2.75], $p<0.05$ ). PER discriminated the left (contralesional) targets in the covert endogenous attention condition less accurately than the right targets; the discrimination accuracy for left targets was similar to chance levels (50\%) while the discrimination accuracy for right targets was above chance levels. In the covert exogenous attention condition, PER showed similar performance in both visual fields, which was higher than the discrimination accuracy at chance levels. 


\subsection{Functional Connectivity at Rest}

\subsubsection{Frontoparietal Functional Connectivity}

The mean correlation strength between the eight frontal seeds and the five parietal regions is shown in Figure 4. Since voxels overlapping with the right parietal lesion were excluded from the analysis, a weak correlation between frontal seeds and right perilesional parietal regions was interpreted as a proximal effect of the structural lesion. The spared voxels of the right SPL and the right ANG were affected by the proximal extension of the lesion. The perilesional activity of the right SPL and the right ANG was weakly correlated with all frontal seeds (not significantly different from zero or negative, see table in figure 4). The perilesional activity of the AAL region, labeled right IPL (corresponding to the IPS), showed weak correlations with the activity of the most dorsal frontal seeds (correlation strengths with the left and right M1s and the right FEF were not significantly different from zero, $p>10^{-6}$ for all correlations) but showed strong correlations with the activity of frontal VAN seeds (MFG and IFG in the right hemisphere). In contrast, the perilesional activity of the right precuneus and the right SMG showed significant correlations with all frontal seeds (all correlation strengths significantly different from zero, $p<10^{-6}$ for all correlations). Furthermore, the strength of the correlation of the right SMG with all attentional and motor seeds (except left M1) was significantly higher than the correlation of their left counterpart ( $p<10^{-6}$ for all correlations).

To summarize, the left FEF was functionally connected to the left IPS/SPL (corresponding to the SPL and IPL regions of the AAL atlas). In contrast, the lesion in the right hemisphere disrupted the functional correlation between the right FEF and the right IPS/SPL. These results were similar to those reported previously [48]. The activity of the IFG and MFG was strongly correlated with that of the SMG within the right hemisphere, despite the presence of a proximal lesion in the other parts of the right parietal cortex. Their activity was also strongly correlated with the IPL (bilaterally) and, surprisingly, with the left intact SPL. These results were also similar to those reported previously [48].

To determine whether the leftward asymmetry of functional connectivity found mainly between the frontal seeds and the SPL was specific to this region, we performed paired t-tests and compared the mean correlation scores between 18 frontal $A A L$ areas of the left hemisphere and seven $A A L$ parietal areas of the left and right hemispheres $(n=126 ; t(125)=-0.27 ; p=0.79)$ to the scores between 18 frontal AAL areas of the right hemisphere and seven AAL parietal areas of the left and right hemispheres $(n=126 ; t(125)=-0.47 ; p=0.64)$. The results confirmed the absence of a global left asymmetry in the frontoparietal functional connectivity and revealed a specific disruption of the functional relationship between the dorsal part of the right posterior parietal cortex and the dorsal frontal seeds.

\subsubsection{Fronto-Occipital Functional Connectivity}

For the fronto-occipital functional connectivity, the underlined values in the table of Figure 5 indicate correlations that are significantly different from zero. Values in bold indicate that the correlation between the seed and the AAL region is significantly different from the correlation between the seed and the AAL region in the other hemisphere.

For the attentional networks (DAN and VAN; Figure 5, - top row and table), we investigated the fronto-occipital functional connectivity by comparing the correlation strengths between the right 
and left hemispheres of all four attentional frontal seeds with the inferior occipital region-, which is involved in attentional processes [6]. The frontal seeds for the VAN, IFG, and MFG of the right hemisphere, were functionally correlated to the left and right inferior occipital region equally (ps > $\left.10^{-6}\right)$. In contrast, the correlation between the right FEF (DAN) and the activity of the left inferior occipital region was stronger than that between the right FEF and the right inferior occipital region $\left(\mathrm{p}<10^{-6}\right)$.

For the motor networks (Figure 5, - bottom row and table), we investigated the fronto-occipital functional connectivity by comparing the correlation scores between the right and left hemispheres of all four motor frontal seeds with the cuneus, - which was the region spared by the lesion and limited by the parieto-occipital sulcus, and includes the human homolog of V6A (associated with reaching) [30]. We found that the correlation between the left and right M1 with the activity of the left cuneus was stronger than the correlation between both $M 1$ with the activity of the right cuneus $\left(p<10^{-6}\right)$; however, no asymmetry was found for the correlations with the left and right SMA ( $p>$ $\left.10^{-6}\right)$.

To summarize, the fronto-occipital connectivity pattern found in this study showed strong associations with the specific behavioral deficit of endogenous attention and arm reaching movements of our patient. We found that 1 ) the right FEF showed significantly stronger correlations with the left inferior occipital region than with the right inferior occipital region, while such strength asymmetries were absent for the correlations with the right MFG or the right IFG, and 2) the M1 regions showed significantly stronger correlations with the left cuneus than with the right cuneus, while such strength asymmetries were absent for the correlations with the left and right SMAs.

\section{Discussion}

The parietal cortex plays an important role in multisensory spatial integration and visuomotor control [50-52], as well as in attentional facilitation by directly interacting with the peripheral field representations of the inferior occipitotemporal cortex [32, 53, 54]. A focal IPS/SPL stroke could therefore explain the concomitant visuomotor and visuo-attentional deficits in our patient PER. Additionally, since the brain is organized into a set of widely distributed and functionally interconnected networks, a focal stroke, - for example, in the IPS/SPL of optic ataxia patients, - could induce proximal and distal dysfunction in structurally intact regions that are functionally related to the damaged area. Optic ataxia has frequently been described as arising from an occipitofrontal disconnection between visual processing systems and motor regions [55-58], and lesions in the parietal cortex were shown to cancel pre-saccadic attentional facilitation; thus, suggesting that the functional relationship between the FEF and V4, characterized in the macaque, might operate via the parietal cortex.

Brain networks related to specific functions, such as attention (DAN/VAN) or sensorimotor integration, can be studied using resting-state fMRI [40-42]. For example, using resting-state fMRI, Corbetta et al. [39] showed that structural damage to the right TPJ (VAN) produces a virtual lesion of the IPS/SPL regions within the DAN in the same (right) hemisphere. Conversely, Gillebert et al. [34] concluded that structural damage to the right IPS/SPL (DAN) has no reciprocal proximal effect on the VAN as it does not affect the strength of the main inter-and intra-hemispheric connectivity of the right TPJ. The results of the present behavioral and resting-state fMRI study of a single case of optic ataxia indicated the absence of reciprocal proximal effects since, in our patient, the activity 
of the SMG (including right TPJ) was highly correlated with right MFG and IFG activity. The two frontal seeds were also highly correlated with the activity in the left and right inferior occipital regions. The activity pattern was found to be well-associated with the ability of PER to orient her attention and respond to peripheral exogenous cues in both the left and right visual fields.

Regarding the behavioral trials, our results were similar to those found by McIntosh et al. [59], i.e., following a right posterior parietal lesion in the IPS/SPL region, the patient showed concomitant and related reaching and perceptual deficits. More specifically, in her left contralesional visual field, patient PER exhibited both reaching and endogenous attention deficits. Consequently, we showed that the IPS/SPL lesion specifically affected the integrity of the dorsal visuomotor and dorsal attentional networks, directly, as well as, via indirect effects on the interaction between M1 and V6A (within the parieto-occipital sulcus, at the anterior limit of the Cuneus), and between FEF and V4 (within Inferior occipital gyrus), respectively. The activity within the dorsal visuomotor and dorsal attention networks was previously assessed in the healthy brain using resting-state fMRI. These are typically described as bilateral networks without any apparent asymmetry [48, 49, 60-64]. This contrasts with the pattern of functional connectivity that we found for the dorsal visuomotor and dorsal attention networks in PER, which were strongly asymmetrical. Specifically, the functional connectivity of the right FEF (DAN) and the left and right M1 seeds (dorsal visuomotor network) were strongly biased toward the left hemisphere, not only for the parietal regions - (because the right IPS/SPL region was structurally damaged) - but also for the ventral and dorsal occipital regions.

Within the attentional networks, the lesion in the right parietal cortex disrupted the integrity of the frontoparietal functional connectivity such that the activity of both the left and right FEF showed stronger correlations with the activity of the intact left IPS/SPL region. This connectivity pattern might explain the patient's bias toward the right hemifield in the letter discrimination task with the endogenous condition. The association between the right FEF and the inferior occipital cortices (that include the human homolog of $\mathrm{V4}, 6$ ) was also asymmetrical, - even though the occipital cortex in both the hemispheres was structurally spared. Previously, electrophysiological studies in monkeys demonstrated a functional link between FEF and V4 during perceptual tasks involving covert shifts in attention [1-4, 6, 8], especially endogenous attention [7]. Thus, the biased functional association of the right FEF (DAN) with the inferior occipital cortex of the left hemisphere in PER could also explain the difference in the patient's discrimination abilities in the letter discrimination task between the two hemifields under endogenous conditions, wherein, PER performed better in the right visual field. Conversely, the patient's performance between hemifields was similar under exogenous conditions, which was probably related to similar correlation strengths between the frontal seeds of the VAN (right IFG and right MFG) and the left and right inferior occipital cortices.

Within the motor network, the lesion in the right parietal cortex disrupted the integrity of the frontoparietal functional connectivity such that the activity of both the left and right M1 showed stronger correlations with the activity in the intact left SPL and ANG. We examined the effect of the parietal lesion on the functional connectivity between the left and right $\mathrm{M} 1$ and the cuneus regions to better understand the neural functional network underlying the direct visuomotor transformations of the reaching behavior, which is typically impaired in optic ataxia (e.g., [22-25]). The cuneus region of the AAL parcellation used in this study was limited anteriorly by the parietooccipital sulcus, which included the putative human homolog of the macaque V6A [30]. Several studies have shown a functional involvement of the posterior dorsomedial occipito-parietal cortex, - labeled SPOC (superior parieto-occipital cortex), - in arm reaching movements (e.g., [25-30, 64, 
65]). Moreover, direct anatomical connections between the dorsal occipito-parietal cortex and the dorsal premotor cortex, - which projects to $\mathrm{M} 1$, - have been identified in monkeys using tracing studies [21]. We found that the activity of the left and right M1 showed a stronger correlation with the activity of the left cuneus compared to the correlation with the activity of the right cuneus, although this region was structurally spared in both the hemispheres. - - We suggest that the behavioral impairment in the reaching behavior of the optic ataxia patient to guide movements toward the left visual field with both hands - (field effect) - could be associated with the weak functional connectivity of the dorsal occipito-parietal areas of the right hemisphere with the regions that control the left and right hands, i.e., - M1 right and M1 left, respectively. The long-range impact of a focal lesion on resting-state functional connectivity measures is an important finding regarding optic ataxia. The localization of a typical lesion, which is more classically tested by lesion superimposition methods and lesion-mapping symptoms, has recently drawn the attention of researchers to the parieto-occipital sulcus instead of the IPS/SPL (e.g., [66]). In this study, we found that PER's lesion was in the IPS/SPL, and the parieto-occipital sulcus was structurally intact. Despite this, the functional network between the cuneus and M1 was biased toward the left hemisphere. This loss of coherence between the M1s and the cuneus in the right hemisphere showed a strong association with the impaired performance of the patient in the left visual field.

In this paper, we demonstrated an asymmetry in resting-state functional connectivity within a patient with a brain lesion. Although our findings were informative, the results could be more accurate and have greater statistical support if a group of healthy, age-matched controls was studied simultaneously with the patient. Future studies could, for example, investigate whether the above-mentioned asymmetries originate from a decrease in intra-hemisphere connectivity in the lesioned hemisphere and/or an increase in interhemispheric connectivity.

\section{Conclusions}

In summary, the results of this study demonstrated that the single-case approach proposed here, - although exploratory and descriptive, - could provide valuable information for future studies that aim to investigate functional connectivity changes underlying behavioral changes after specific structural lesions. These results were consistent with those of a previous study, where it was shown that behavioral deficits were intrinsically related to a loss of coherence in the spontaneous activity of different functional networks when measured at rest [67]. Measuring the activity within the resting-state networks is an effective method to obtain information about potential proximal and distal consequences of the damage to the brain [47]. Finally, due to the uniqueness of lesions, as well as, their impact on the functional reconfiguration of brain networks, the single-case approach could be applied to better characterize structure-function relationships [68].

\section{Author Contributions}

Conceptualisation: FHB and LP; Resources and testing: GR, LP and FC; Analysis: CG, EMA, FC, KR, FHB; Writing initial: EMA and CG; Writing final: LP, FHB, KR.

\section{Competing Interests}

The authors have declared that no competing interests exist. 


\section{References}

1. Moore T, Armstrong KM. Selective gating of visual signals by microstimulation of frontal cortex. Nature. 2003; 421: 370-373.

2. Moore T, Fallah M. Microstimulation of the frontal eye field and its effects on covert spatial attention. J Neurophysiol. 2004; 91: 152-162.

3. Armstrong KM, Fitzgerald JK, Moore T. Changes in visual receptive fields with microstimulation of frontal cortex. Neuron. 2006; 50: 791-798.

4. Armstrong KM, Moore T. Rapid enhancement of visual cortical response discriminability by microstimulation of the frontal eye field. Proc Natl Acad Sci USA. 2007; 104: 9499-9504.

5. Neggers SF, Huijbers W, Vrijlandt CM, Vlaskamp BN, Schutter DJ, Kenemans JL. TMS pulses on the frontal eye fields break coupling between visuospatial attention and eye movements. J Neurophysiol. 2007; 98: 2765-2778.

6. Sylvester CM, Shulman GL, Jack Al, Corbetta M. Asymmetry of anticipatory activity in visual cortex predicts the locus of attention and perception. J Neurosci. 2007; 27: 14424-14433.

7. Schafer RJ, Moore T. Selective attention from voluntary control of neurons in prefrontal cortex. Science. 2011; 332: 1568-1571.

8. Hu M, Clark KL, Gong X, Noudoost B, Li M, Moore T, et al. Copula regression analysis of simultaneously recorded frontal eye field and inferotemporal spiking activity during objectbased working memory. J Neurosci. 2015; 35: 8745-8757.

9. Corbetta M, Patel G, Shulman GL. The reorienting system of the human brain: From environment to theory of mind. Neuron. 2008; 58: 306-324.

10. Corbetta M, Shulman GL. Spatial neglect and attention networks. Annu Rev Neurosci. 2011; 34: 569-599.

11. Vallar G, Perani D. The anatomy of unilateral neglect after right-hemisphere stroke lesions. A clinical/CT-scan correlation study in man. Neuropsychologia. 1986; 24: 609-622.

12. Mort DJ, Malhotra P, Mannan SK, Rorden C, Pambakian A, Kennard C, et al. The anatomy of visual neglect. Brain. 2003; 126: 1986-1997.

13. Jeannerod M. Mechanisms of visuomotor coordination: A study in normal and brain-damaged subjects. Neuropsychologia. 1986;24(1):41-78.

14. Perenin MT, Vighetto A. Optic ataxia: A specific disruption in visuomotor mechanisms. I. Different aspects of the deficit in reaching for objects. Brain. 1988; 111: 643-674.

15. Milner AD, Goodale MA. The visual brain in action. 2nd ed. New York: Oxford University Press; 2006.

16. Khan AZ, Pisella L, Vighetto A, Cotton F, Luauté J, Boisson D, et al. Optic ataxia errors depend on remapped, not viewed, target location. Nat Neurosci. 2005; 8: 418-420.

17. Milner AD, Goodale MA. Two visual systems re-viewed. Neuropsychologia. 2008; 46: 774-785.

18. Blangero A, Khan A, Rode $G$, Rossetti $Y$, Pisella L. Dissociation between intentional and automatic remapping: Different levels of inter-hemispheric transfer. Vision Res. 2011; 51: 932939.

19. Granek JA, Pisella L, Blangero A, Rossetti Y, Sergio LE. The role of the caudal superior parietal lobule in updating hand location in peripheral vision: Further evidence from optic ataxia. PLoS One. 2012; 7: e46619. 
20. Vindras $P$, Blangero $A$, Ota $H$, Reilly $K T$, Rossetti $Y$, Pisella $L$. The pointing errors in optic ataxia reveal the role of "peripheral magnification" of the PPC. Front Integr Neurosci. 2016; 10: 27.

21. Tanné J, Boussaoud D, Boyer-Zeller N, Rouiller EM. Direct visual pathways for reaching movements in the macaque monkey. Neuroreport. 1995; 7: 267-272.

22. Prado J, Clavagnier S, Otzenberger $\mathrm{H}$, Scheiber $\mathrm{C}$, Kennedy H, Perenin $\mathrm{M}-\mathrm{T}$. Two cortical systems for reaching in central and peripheral vision. Neuron. 2005; 48: 849-858.

23. Galletti C, Kutz DF, Gamberini M, Breveglieri R, Fattori P. Role of the medial parieto-occipital cortex in the control of reaching and grasping movements. Exp Brain Res. 2003; 153: 158-170.

24. Pisella L, Binkofski F, Lasek K, Toni I, Rossetti Y. No double-dissociation between optic ataxia and visual agnosia: Multiple sub-streams for multiple visuo-manual integrations. Neuropsychologia. 2006; 44: 2734-2748.

25. Vesia M, Prime SL, Yan X, Sergio LE, Crawford JD. Specificity of human parietal saccade and reach regions during transcranial magnetic stimulation. J Neurosci. 2010; 30: 13053-13065.

26. Filimon F, Nelson JD, Hagler DJ, Sereno MI. Human cortical representations for reaching: Mirror neurons for execution, observation, and imagery. Neuroimage. 2007; 37: 1315-1328.

27. Filimon F, Nelson JD, Huang R-S, Sereno MI. Multiple parietal reach regions in humans: Cortical representations for visual and proprioceptive feedback during on-line reaching. J Neurosci. 2009; 29: 2961-2971.

28. Cavina-Pratesi C, Monaco S, Fattori P, Galletti C, McAdam TD, Quinlan DJ, et al. Functional magnetic resonance imaging reveals the neural substrates of arm transport and grip formation in reach-to-grasp actions in humans. J Neurosci. 2010; 30: 10306-10323.

29. Galati G, Committeri G, Pitzalis S, Pelle G, Patria F, Fattori P, et al. Intentional signals during saccadic and reaching delays in the human posterior parietal cortex. Eur J Neurosci. 2011; 34: 1871-1885.

30. Pitzalis S, Sereno MI, Committeri G, Fattori P, Galati G, Tosoni A, et al. The human homologue of macaque area V6A. Neuroimage. 2013; 82: 517-530.

31. Striemer C, Blangero A, Rossetti Y, Boisson D, Rode G, Vighetto A, et al. Deficits in peripheral visual attention in patients with optic ataxia. Neuroreport. 2007; 18: 1171-1175.

32. Blangero A, Khan AZ, Salemme R, Deubel H, Schneider WX, Rode G, et al. Pre-saccadic perceptual facilitation can occur without covert orienting of attention. Cortex. 2010; 46: 11321137.

33. Shomstein S, Lee J, Behrmann M. Top-down and bottom-up attentional guidance: Investigating the role of the dorsal and ventral parietal cortices. Exp Brain Res. 2010; 206: 197-208.

34. Gillebert CR, Mantini D, Thijs V, Sunaert S, Dupont $P$, Vandenberghe R. Lesion evidence for the critical role of the intraparietal sulcus in spatial attention. Brain. 2011; 134: 1694-1709.

35. Pisella L, André V, Gavault E, Le Flem A, Luc-Pupat E, Glissoux C, et al. A test revealing the slow acquisition and the dorsal stream substrate of visuo-spatial perception. Neuropsychologia. 2013; 51: 106-113.

36. Chica $A B$, Bartolomeo $P$, Lupiáñez J. Two cognitive and neural systems for endogenous and exogenous spatial attention. Behav Brain Res. 2013; 237: 107-123.

37. Cubelli R, De Bastiani P. 150 Years after Leborgne: Why is Paul Broca so important in the history of neuropsychology? Cortex. 2010; 47: 146-147.

38. Ramsey LE, Siegel JS, Baldassarre A, Metcalf NV, Zinn K, Shulman GL, et al. Normalization of network connectivity in hemispatial neglect recovery. Ann Neurol. 2016; 80: 127-141. 
39. Corbetta M, Kincade MJ, Lewis C, Snyder AZ, Sapir A. Neural basis and recovery of spatial attention deficits in spatial neglect. Nat Neurosci. 2005; 8: 1603-1610.

40. Power JD, Cohen AL, Nelson SM, Wig GS, Barnes KA, Church JA, et al. Functional network organization of the human brain. Neuron. 2011; 72: 665-678.

41. Yeo BT, Krienen FM, Sepulcre J, Sabuncu MR, Lashkari D, Hollinshead M, et al. The organization of the human cerebral cortex estimated by intrinsic functional connectivity. J Neurophysiol. 2011; 106: 1125-1165.

42. Hacker CD, Laumann TO, Szrama NP, Baldassarre A, Snyder AZ, Leuthardt EC, et al. Resting state network estimation in individual subjects. Neuroimage. 2013; 82: 616-633.

43. Corbetta M, Shulman GL. Control of goal-directed and stimulus-driven attention in the brain. Nat Rev Neurosci. 2002; 3: 201-215.

44. Tzourio-Mazoyer N, Landeau B, Papathanassiou D, Crivello F, Etard O, Delcroix N, et al. Automated anatomical labeling of activations in SPM using a macroscopic anatomical parcellation of the MNI MRI single-subject brain. Neurolmage. 2002; 15: 273-289.

45. Chica $A B$, Martín-Arévalo $E$, Botta $F$, Lupiáñez J. The spatial orienting paradigm: How to design and interpret spatial attention experiments. Neurosci Biobehav Rev. 2014; 40: 35-51.

46. Cox RW. AFNI: Software for analysis and visualization of functional magnetic resonance neuroimages. Comput Biomed Res. 1996; 29: 162-173.

47. Zhang $Y$, Brad M, Smith S. Segmentation of brain MR images through a hidden Markov random field model and the expectation-maximization algorithm. IEEE Trans Med Imag. 2001; 20: 4557.

48. Fox MD, Corbetta M, Snyder AZ, Vincent JL, Raichle ME. Spontaneous neuronal activity distinguishes human dorsal and ventral attention systems. Proc Natl Acad Sci USA. 2006; 103: 10046-10051.

49. Grefkes C, Wang LE, Eickhoff SB, Fink GR. Noradrenergic modulation of cortical networks engaged in visuomotor processing. Cereb Cortex. 2010; 20: 783-797.

50. De Renzi E. Balint-Holmes' Syndrome. In: Classic cases in neuropsychology: Vol 1. Hove, East Sussex: Psychology Press; 1996.

51. Andersen RA, Andersen KN, Hwang EJ, Hauschild M. Optic ataxia: From Balint's syndrome to the parietal reach region. Neuron. 2014; 81: 967-983.

52. Gaveau V, Pélisson D, Blangero A, Urquizar C, Prablanc C, Vighetto A, et al. Saccade control and eye-hand coordination in optic ataxia. Neuropsychologia. 2008; 46: 475-486.

53. Ungerleider LG, Galkin TW, Desimone R, Gattass R. Cortical connections of area V4 in the macaque. Cereb Cortex. 2008; 18: 477-499.

54. Janssen $P$, Verhoef B-E, Premereur E. Functional interactions between the macaque dorsal and ventral visual pathways during three-dimensional object vision. Cortex. 2018; 98: 218-227.

55. Bálint R. Seelenlähmung des "Schauens", optische Ataxie, räumliche Störung der Aufmerksamkeit. pp. 67-81. Eur Neurol. 1909; 25: 67-81.

56. Garcin R, Rondot P, de Recondo J. Optic ataxia localized in 2 left homonymous visual hemifields (clinical study with film presentation). Rev Neurol (Paris). 1967; 116: 707-714.

57. Pierrot-Deseilligny C, Gray F, Brunet P. Infarcts of both inferior parietal lobules with impairment of visually guided eye movements, peripheral visual inattention and optic ataxia. Brain. 1986; 109: 81-97. 
58. Gaymard B, Rivaud S, Rigolet MH, Pierrot-Deseilligny C. Bilateral crossed optic ataxia in a corpus callosum lesion. J Neurol Neurosurg Psychiatry. 1993; 56: 323-324.

59. McIntosh RD, Mulroue A, Blangero A, Pisella L, Rossetti Y. Correlated deficits of perception and action in optic ataxia. Neuropsychologia. 2011; 49: 131-137.

60. Bressler SL, Tang W, Sylvester CM, Shulman GL, Corbetta M. Top-down control of human visual cortex by frontal and parietal cortex in anticipatory visual spatial attention. J Neurosci. 2008; 28: 10056-10061.

61. Sylvester CM, Jack Al, Corbetta M, Shulman GL. Anticipatory suppression of nonattended locations in visual cortex marks target location and predicts perception. J Neurosci. 2008; 28: 6549-6556.

62. Gallivan JP, McLean DA, Valyear KF, Pettypiece CE, Culham JC. Decoding action intentions from preparatory brain activity in human parieto-frontal networks. J Neurosci. 2011; 31: 9599-9610.

63. Otten ML, Mikell CB, Youngerman BE, Liston C, Sisti MB, Bruce JN, et al. Motor deficits correlate with resting state motor network connectivity in patients with brain tumours. Brain. 2012; 135: 1017-1026.

64. Matt E, Foki T, Fischmeister F, Pirker W, Haubenberger D, Rath J, et al. Early dysfunctions of fronto-parietal praxis networks in Parkinson's disease. Brain Imaging Behav. 2017; 11: 512-525.

65. Rossit S, McAdam T, McLean DA, Goodale MA, Culham JC. fMRI reveals a lower visual field preference for hand actions in human superior parieto-occipital cortex (SPOC) and precuneus. Cortex. 2013; 49: 2525-2541.

66. Karnath $\mathrm{H}-\mathrm{O}$, Perenin M-T. Cortical control of visually guided reaching: Evidence from patients with optic ataxia. Cereb Cortex. 2005; 15: 1561-1569.

67. Carter AR, Astafiev SV, Lang CE, Connor LT, Rengachary J, Strube MJ, et al. Resting interhemispheric functional magnetic resonance imaging connectivity predicts performance after stroke. Ann Neurol. 2010; 67: 365-375. DOI: 10.1002/ana.21905

68. Zhang D, Raichle ME. Disease and the brain's dark energy. Nature Review Neurology. 2010; 6: $15-28$.

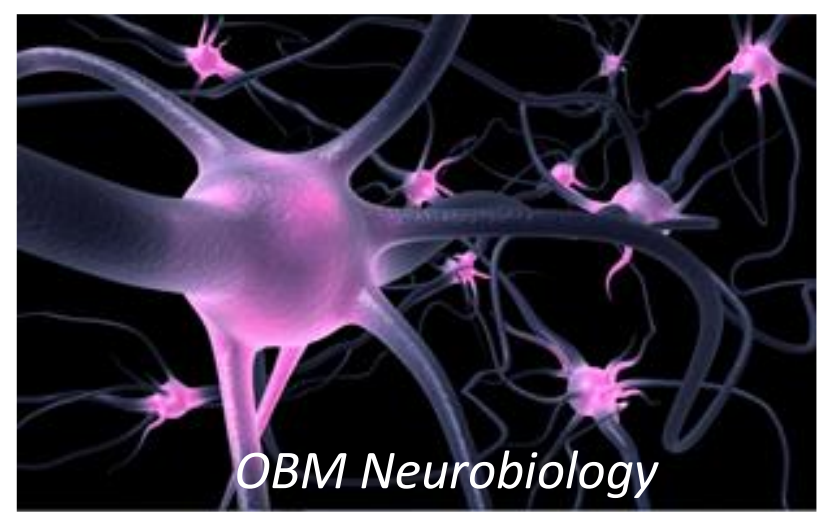

Enjoy OBM Neurobiology by:

1. Submitting a manuscript

2. Joining volunteer reviewer bank

3. Joining Editorial Board

4. Guest editing a special issue

For more details, please visit:

http://www.lidsen.com/journals/neurobiology 